\title{
Oxidized Low-Density Lipoprotein Antigen Transport Induces Autoimmunity in the Renal Tubulointerstitium
}

\author{
Bancha Satirapoj ${ }^{\text {a, d }}$ Kevin W. Bruhn ${ }^{a}$ Cynthia C. Nast ${ }^{a, c}$ Ying Wang ${ }^{a}$ \\ Tiane Dai $^{\mathrm{a}}$ Janine LaPage ${ }^{\mathrm{a}} \quad$ Xiwei Wu $^{\mathrm{b}}$ Rama Natarajan $^{\mathrm{b}}$ Sharon G. Adler ${ }^{\mathrm{a}}$ \\ ${ }^{a}$ Harbor-UCLA Los Angeles Biomedical Research Institute, Torrance, Calif., ${ }^{b}$ Beckman Research Institute of City \\ of Hope, Duarte, Calif., and ${ }^{\mathrm{C} C e d a r s-S i n a i ~ M e d i c a l ~ C e n t e r, ~ L o s ~ A n g e l e s, ~ C a l i f ., ~ U S A ; ~}{ }^{\mathrm{d} D i v i s i o n}$ of Nephrology, \\ Phramongkutklao Hospital and College of Medicine, Bangkok, Thailand
}

\section{Key Words}

Oxidized low-density lipoprotein · Chronic kidney disease •

Renal autoimmunity $\cdot$ Tubulointerstitial inflammation

\begin{abstract}
Background/Aims: Chronic kidney disease involves inflammation/oxidative stress, which contributes to progressive kidney injury. Methods: Male Sprague-Dawley rats underwent 5/6 nephrectomy $(\mathrm{Nx})$ or sham $\mathrm{Nx}$ and were sacrificed after 2 days, 2 weeks and 4 weeks. Microarray analysis expression sets over time suggested the evolution of renal lymphocyte infiltration and antigen-presenting cell (APC) activation after 5/6Nx. RT-PCR analysis also confirmed the migration and activation of lymphocytes and APCs through the upregulation of CD3, CXCR3/CXCL10 and CCR7/CCL19 mRNA in remnant kidney (RK). Purified T lymphocytes from spleen and unilateral ureteral obstruction (UUO) kidney were incubated with oxidized low-density lipoprotein (Ox-LDL)-treated major histocompatibility complex class II (MHC II)-expressing APCs. Culture supernatant was collected for mouse IFN- $\gamma$ ELISA and cell proliferation was measured. Results:
\end{abstract}

Ox-LDL deposited predominantly in renal tubulointerstitial areas of RK, increased over time, and co-stained with lectinlike Ox-LDL receptor in affected renal tubular cells. Both OxLDL and renal-specific glycoprotein Tamm-Horsfall protein were identified in renal lymph nodes. Cells co-staining for major MHC II and Ox-LDL were observed in RK and draining renal lymph nodes after 5/6Nx. Similarly, Ox-LDL was also present in tubules after UUO, CD3-positive T cells were present in the interstitium, and Ox-LDL-treated MHC II-expressing APCs induced proliferation and IFN- $\gamma$ production in renal tubulointerstitial T lymphocytes isolated from kidneys after UUO. Conclusions: These data demonstrate that the tubulointerstitial inflammatory infiltrate that accompanies chronic kidney disease reflects, at least in part, the development of autoimmunity to novel antigens generated during renal injury.

Copyright $\odot 2012$ S. Karger AG, Basel

The results presented in this paper have not been published previously in whole or part, except in abstract format.

\section{KARGER}

Fax +41613061234 E-Mail karger@karger.ch www.karger.com
(C) 2012 S. Karger AG, Basel

0250-8095/12/0356-0520\$38.00/0

Accessible online at:

www.karger.com/ajn
Dr. Sharon G. Adler

Division of Nephrology and Hypertension

Los Angeles Biomedical Research Center

1124 West Carson Street, Torrance, CA 90502 (USA)

Tel. +1 310222 3891, E-Mail sadler@ labiomed.org 
Table 1. Real-time RT-PCR primer sequences

\begin{tabular}{lllr}
\hline Gene & Forward primer & Reverse primer & Size, bp \\
\hline 18 S & GGACAGGATTGACAGATTGATAG & TCGTTATCGGAATTAACCAGACAA & 103 \\
CD3 & TTACCGAATGTGCCAGAACTGT & TCATGGCCTGCAAAGCAGTA & 120 \\
CXCR3 & GAGGTCAGTGAACGTCAAGTGCTA & TCGCTTTCGTTTCCCCATA & 95 \\
CXCL10 & ATCCTGTCCGCATGTTGAGATC & TGGCTCACCGCTTTCAATAAG & 108 \\
CCR7 & GGAAGCCCACGAAAAACGT & TGGTGTTCTCGCCGATGTAGT & 102 \\
CCL19 & GCATCATCCGAAGACTGAAGAAGT & CTCACGTTCACACCGACTCTCTA & 197 \\
\hline
\end{tabular}

\section{Background}

The renal interstitium in chronic kidney disease (CKD) expresses features of lymphoid neogenesis, a characteristic of tissue injury observed whenever inflammatory and immune responses are unable to eradicate antigen, as would be anticipated when the antigen is endogenous [1]. In such conditions, 'steady-state' migratory antigen-presenting cells (APCs), capable of activation and migration, facilitate the transport of antigens from non-lymphatic organs to draining lymph nodes for presentation and activation of naive T lymphocytes [2-4]. This observation confers plausibility to the hypothesis that the activated immune cells in the injured renal tubulointerstitium may be derived from the amplification of naive $\mathrm{T}$ lymphocytes in renal lymph nodes (RLNs) after interaction with APCs bearing endogenous renal antigens [5].

Lipid abnormalities are commonly observed in kidney diseases [6] and, in particular, oxidized low-density lipoprotein (Ox-LDL) has been implicated in the pathogenesis of glomerulosclerosis and tubulointerstitial damage [7-9]. In addition, previous data strongly suggested that $\mathrm{T}$ lymphocytes recognized Ox-LDL as an antigen within the context of major histocompatibility complex (MHC) restriction [10]. Ox-LDL enhanced T-cell proliferation which was inhibited by anti-MHC class II-directed antibodies [11].

A recently published study demonstrated that after 5/6 nephrectomy $(5 / 6 \mathrm{Nx})$, tubular lipid accumulation is associated with upregulation of pathways involved in the tubular reabsorption of filtered lipoproteins and downregulation of pathways involved in lipoprotein and fatty acid metabolism [12]. We therefore investigated whether, after $5 / 6 \mathrm{Nx}$, renal tubular Ox-LDL could behave as a neoantigen. The first step of this process would involve antigen transport from the injured kidney by APCs to draining RLNs. Terminal steps would involve the return of activated $\mathrm{T}$ lymphocytes to the injured renal tissue and activation of autoimmunity in the kidney.

\section{Methods}

\section{Experimental Protocol}

Male Sprague-Dawley rats $(\mathrm{n}=56)$ underwent $5 / 6 \mathrm{Nx}(\mathrm{n}=27)$ by unilateral $\mathrm{Nx}$ and ligation of two-thirds of the vessels to the contralateral kidney or sham Nx. Rats were sacrificed 2 days, 2 weeks, and 4 weeks after surgery. C57BL/6 mice were subjected to left kidney unilateral ureteral obstruction (UUO). At 14 days, renal tissue was harvested and renal $\mathrm{T}$ lymphocytes were extracted and purified using a mouse T-lymphocyte enrichment set (Becton Dickinson, San Jose, Calif., USA).

\section{Gene Array Analysis}

Microarray Gene Set Enrichment Analysis (GSEA; Broad Institute, Cambridge, Mass., USA) was used to compare data from Affymetrix Gene Chip 230_2 transcription profiles in normal and remnant kidneys (RK) 2 days, 2 weeks and 4 weeks after $5 / 6 \mathrm{Nx}$. Sequences used in the design of the Gene Chip Rat Genome 2302.0 arrays were selected from publicly available (GenBank $^{\circledR}, \mathrm{dbEST}$, and RefSeq), well-annotated mRNA sequences. Total RNA from $3 \mathrm{RK}$ at each time point and 3 normal kidneys were labeled and hybridized to Affymetrix Gene Chips. Data were expressed as the average differences between the perfect match and mismatch probes.

\section{Real-Time RT-PCR Analysis}

Total RNA was isolated from normal control kidneys and RK 2 days, 2 weeks and 4 weeks after $5 / 6 \mathrm{Nx}$ using RNA STAT- $60^{\mathrm{TM}}$ (Tel-Test Inc., Tex., USA). Real-time RT-PCR was performed with specific primers in table 1. All PCRs were run in an ABI 7900 Sequence Detection System (Applied Biosystems). Raw data consisted of the PCR cycle number required to reach a fluorescence threshold $\left(\mathrm{C}_{\mathrm{t}}\right)$. Values were normalized to $18 \mathrm{~S}$ and calculated using the comparative $\mathrm{C}_{\mathrm{t}}$ method.

\section{Renal Morphology and Immunohistochemistry}

For light microscopy, kidneys and RLNs were fixed in $10 \%$ buffered formalin, dehydrated and embedded in paraffin. $4-\mu \mathrm{m} \mathrm{sec-}$ tions were stained with periodic acid-Schiff (PAS) reagent for routine histology. For immunohistologic studies, sections were blocked with endogenous enzyme block solution (Dako, Carpinteria, Calif., USA), washed in phosphate-buffered saline, and incubated with primary antibody overnight at $4^{\circ} \mathrm{C}$. The following primary antibodies were used: anti-rabbit polyclonal CD3 for T lymphocytes (Sigma, St. Louis, Mo., USA, 1:300); mouse monoclonal 
Table 2. Microarray GSEA shows an evolutionary process of immune T lymphocytes and APC activation in the RK model

\begin{tabular}{lllll}
\hline Gene sets & $\begin{array}{l}\text { False dis- } \\
\text { covery } \\
\text { rate }\end{array}$ & $\begin{array}{l}2 \\
\text { days }\end{array}$ & $\begin{array}{l}2 \\
\text { weeks }\end{array}$ & $\begin{array}{l}\text { weeks } \\
\text { Hematopoietic cell lineage markers }\end{array}$ \\
\hline Cytokine-cytokine receptor interactive & $<1.6 \%$ & $\uparrow$ & $\uparrow$ & $\uparrow$ \\
Leukocyte transendothelial migration & $<1.6 \%$ & $\uparrow$ & $\uparrow$ & $\uparrow$ \\
IL-2 receptor $\beta$ chain T-cell activation & $4.8 \%$ & $\leftrightarrow$ & $\uparrow$ & $\leftrightarrow$ \\
Natural killer cell-mediated cytotoxicity & $<3.1 \%$ & $\leftrightarrow$ & $\uparrow$ & $\uparrow$ \\
T-cell receptor signaling pathway & $<3.9 \%$ & $\leftrightarrow$ & $\uparrow$ & $\uparrow$ \\
T cytotoxic cell surface molecules & $<3.3 \%$ & $\leftrightarrow$ & $\uparrow$ & $\uparrow$ \\
Cytotoxic T-cell-mediated pathway & $<2.4 \%$ & $\leftrightarrow$ & $\uparrow$ & $\uparrow$ \\
Antigen processing and presentation & $3.4 \%$ & $\leftrightarrow$ & $\leftrightarrow$ & $\uparrow$ \\
\hline
\end{tabular}

antibody EO6 (1:2,000) for Ox-LDL (a generous gift from Dr. Joseph L. Witztum, University of California San Diego, Calif., USA); rabbit polyclonal anti-lectin-like Ox-LDL receptor (LOX-1) (LifeSpan Biosciences, Seattle, Wash., USA, 1:100), and rabbit polyclonal anti-Tamm-Horsfall protein (THP) (Santa Cruz Biotechnology, Santa Cruz, Calif., USA, 1:100). Dextran polymer conjugated with horseradish peroxidase and affinity isolated immunoglobulin (Dako) or peroxidase-conjugated goat anti-mouse IgG+IgM (Jackson ImmunoResearch, West Grove, Pa., USA, 1:500) were used as secondary antibodies. Immunoreactivity was detected by treatment with diaminobenzidine. For quantitation of the interstitial T-lymphocyte cell infiltrate, 10 cortical high-power fields (HPF, $\times 400$ ) per kidney were selected randomly by a blinded observer and the mean cell counts were expressed as cells per HPF.

\section{Immunofluorescence}

At varying time points after $5 / 6 \mathrm{Nx}$, RLN tissue was removed, placed in embedding medium (OCT compound), and frozen with dry ice. $2-\mu \mathrm{m}$ acetone-fixed cryosections were double labeled with primary mouse monoclonal antibody EO6 $(1: 2,000)$ and FITC-conjugated monoclonal MHC II antibody (Abcam, Cambridge, Mass., USA, 1:50). Indirect primary antibody was followed with goat anti-mouse IgG+IgM conjugated to Texas Red (Jackson ImmunoResearch, 1:200). Cell nuclei were counterstained with 4,6-diamidino-2-phenylindole (DAPI).

\section{Purification of T lymphocytes}

Spleens of UUO mice were homogenized with a syringe plunger, while UUO kidneys were minced with a razor blade, then incubated for $45 \mathrm{~min}$ at $37^{\circ} \mathrm{C}$ in DMEM containing $1 \mathrm{mg} / \mathrm{ml}$ collagenase D (Roche) and $0.2 \mathrm{mg} / \mathrm{ml}$ DNAase I. T cells were purified using a mouse T-lymphocyte enrichment set (Becton Dickinson). Magnetic bead separation was performed according to the manufacturer's instructions. Purified T cells from the spleen and UUO kidney were incubated with FITC-conjugated CD3 antibody for 30 min, washed with staining buffer, and analyzed by flow cytometry.

T-Cell Proliferation and Stimulation Assay

LDL was purified to homogeneity by ultracentrifugation and oxidized using $20 \mu \mathrm{M}$ cupric sulfate in PBS at $37^{\circ} \mathrm{C}$ for $24 \mathrm{~h}$ (ob- tained from GenWay Biotech, San Diego, Calif., USA). The level of oxidation was measured using a TBARS (thiobarbituric acid reactive substances) determination using a malondialdehyde standard. All lipoprotein preparations were tested for endotoxin (assay kit, Sigma). RAW264.7 cells purchased from the ATCC were used as stimulators. RAW264.7 cells were cultured for $48 \mathrm{~h}$ in the absence or presence of $\mathrm{Ox}$-LDL at varying concentrations and then treated with mitomycin c $(50 \mu \mathrm{g} / \mathrm{ml})$ for $1 \mathrm{~h}$ in 96 -well tissue culture plates. Afterwards, a total of $1 \times 10^{5} \mathrm{~T}$ cells from the renal tubulointerstitium or spleen were added as responders at a ratio of $1: 10$ (RAW264.7:T cells). After 5 days, $100 \mu$ l of culture supernatant was collected for mouse IFN- $\gamma$ ELISA (Abcam) and $20 \mu$ l diluted BrdU label was added during the last $18 \mathrm{~h}$ of culture. Cell proliferation was measured using a commercially available assay (BrdU Cell Proliferation ELISA Kit; Chemicon, Temecula, Calif., USA).

Phenotypic Analysis of RAW264.7 Cells

RAW264.7 cells untreated or treated with OX-LDL after $48 \mathrm{~h}$ were stained with anti-mouse MHC class II. Photographs were taken with a digital camera at high magnification $(\times 40)$ under a fluorescence microscope. At least 8 horizontal and 8 vertical fields per coverslip were randomly selected. The percentage of MHC IIpositive RAW264.7 cells was calculated using Image-Pro Plus software.

\section{Statistical Analysis}

The results are presented as the mean \pm SD. Statistical analyses of the data were performed by Student's t test and ANOVA and significance for all tests was set at $\mathrm{p}<0.05$.

\section{Results}

\section{Identification of T-Cell and APC Activation by Microarray GSEA following Renal Injury in the RK Model}

Microarray analysis was analyzed using the GSEA tool of the Broad Institute. GSEA showed the evolution of lymphocyte and APC activation after 5/6Nx. 400 biological pathways (gene sets) were analyzed. 47 gene sets at day 2, 111 at week 2 , and 97 at week 4 were significant at false discovery rates $<25 \%$ (corrected for multiple comparisons). This work focuses on further evaluation of selected members of the highly significant gene sets that demonstrate lymphocyte and APC activation in the RK after $5 / 6 \mathrm{Nx}$. Summarized in table 2 , the data show an evolutionary process of immune activation in $5 / 6 \mathrm{Nx}$, with initial immune cell accumulation followed by $\mathrm{T}$-cell and APC activation.

\section{Increased mRNA Expression of CD3 Lymphocytic Chemokine Receptor/Ligand Pairs, CXCR3/CXCL10 and CCR7/CCL19, in RK}

GSEA showed that mRNA expression for the lymphocyte marker CD3 was significantly upregulated in the RK. 


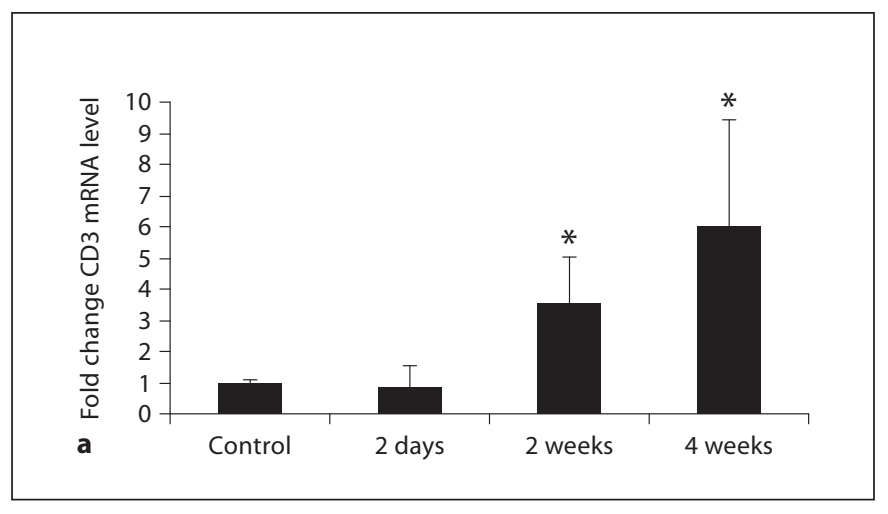

Fig. 1. Increased renal mRNA encoding for CD3, CXCR3/ CXCL10, and CCR7/CCL19 after 5/6Nx. a Renal CD3 mRNA expression significantly increased at 2 weeks and 4 weeks after $5 / 6 \mathrm{Nx}$ compared to normal kidney tissue in samples in which the infarct tissue was excised. b Renal CXCR3 mRNA expression significantly increased at week 4 by 2.6 times, and renal CXCL10 mRNA expression also significantly increased at day 2 , week 2 and week 4 by 1.7-2.0 times compared with that in the control group. c Renal CCR7/CCL19 mRNA expression significantly increased at 2 and 4 weeks after 5/6Nx compared to normal kidney tissue. The expression of $18 \mathrm{~S}$ was used as an internal control. Results are given as mean \pm SD from nine RK and nine control kidney RNA samples at each time point. ${ }^{*} \mathrm{p}<0.05$ vs. control group.

Real-time RT-PCR to confirm the microarray data showed that CD3 lymphocyte mRNA expression was significantly upregulated by 3.5 and 6.0 times in the RK at 2 and 4 weeks, respectively, compared with controls (fig. 1a). These data are consistent with the incremental accumulation of $\mathrm{T}$ cells in the kidney with time after $5 / 6 \mathrm{Nx}$. In addition, mRNA expression of the CXCR3/CXCL10 receptor-ligand pair involved in the recruitment of lymphocytes to sites of inflammation was measured. GSEA showed that mRNA for the CXCR3 chemokine receptor and ligand (CXCL10) for lymphocytes was significantly upregulated in the RK. Real-time RT-PCR of CXCR3 mRNA expression in the $\mathrm{RK}$ at week 4 was significantly upregulated in the RK: 2.6-fold ( $\mathrm{p}<0.05)$ and RT-PCR expression of CXCL10 mRNA expression in the RK at day 2 , week 2 and week 4 was also upregulated by 1.7-2.0 times compared with that in the control group $(\mathrm{p}<0.05)$ (fig. 1b).

APC migration can be triggered by antigen stimuli and requires the chemokine receptor CCR7 [13]. CCR7/ CCL19 mRNA expression, which participates in the migration of mature APCs to lymphoid tissues, was also increased at week 2 and week 4 after $5 / 6 \mathrm{Nx}$ compared to normal kidney (fig. 1c).

Ox-LDL Induces Renal Autoimmunity
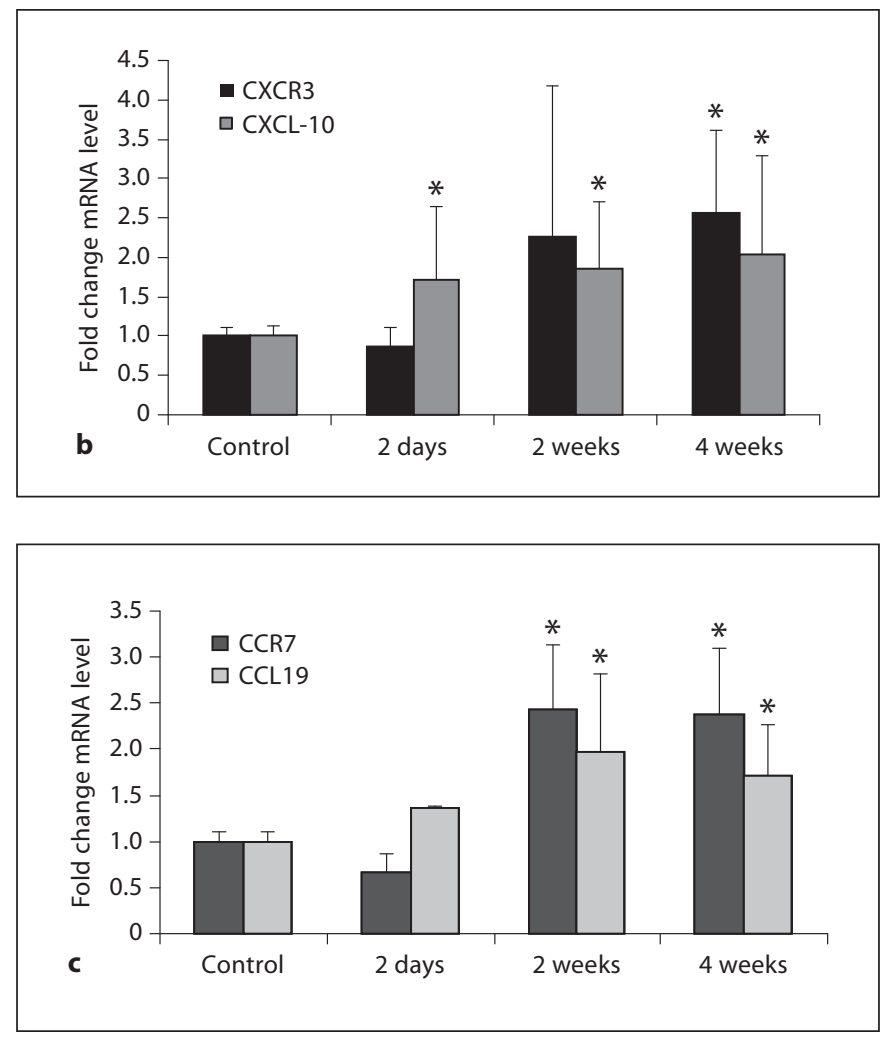

Histopathologic Changes in RK after 5/6Nx

Tubulointerstitial damage after $5 / 6 \mathrm{Nx}$ increased progressively from day 2 to week 4 . Two days after surgery, the RK (infarct included) showed foci of infarcted and nonischemic tissue, with intervening ischemia-reperfusion injury in the bordering renal parenchyma. Injured areas demonstrated a prominent tubulointerstitial inflammatory infiltrate. At 2 weeks, the RK was hypertrophied, with continued tubulointerstitial inflammation. By 4 weeks, glomerulosclerosis and tubulointerstitial fibrosis were prominent, with tubular atrophy and dropout, and continued tubulointerstitial inflammation (fig. 2a).

\section{Accumulation of Interstitial Lymphocytes in RK \\ Tissues}

Increasing numbers of infiltrating CD3+ leukocytes were identified in the cortical interstitium at 2 days, 2 weeks, and 4 weeks after $5 / 6 \mathrm{Nx}$ by immunohistochemical staining. At all time points, CD3+ T lymphocytes could be detected in the mononuclear cell infiltrate. There was also evidence of tubulitis in the RK at week 4 . The interstitial influx of lymphocytes increased progressively from week $2(5.3 \pm 3.8$ cells/HPF $)$ to week $4(17.4 \pm 10.6$ 

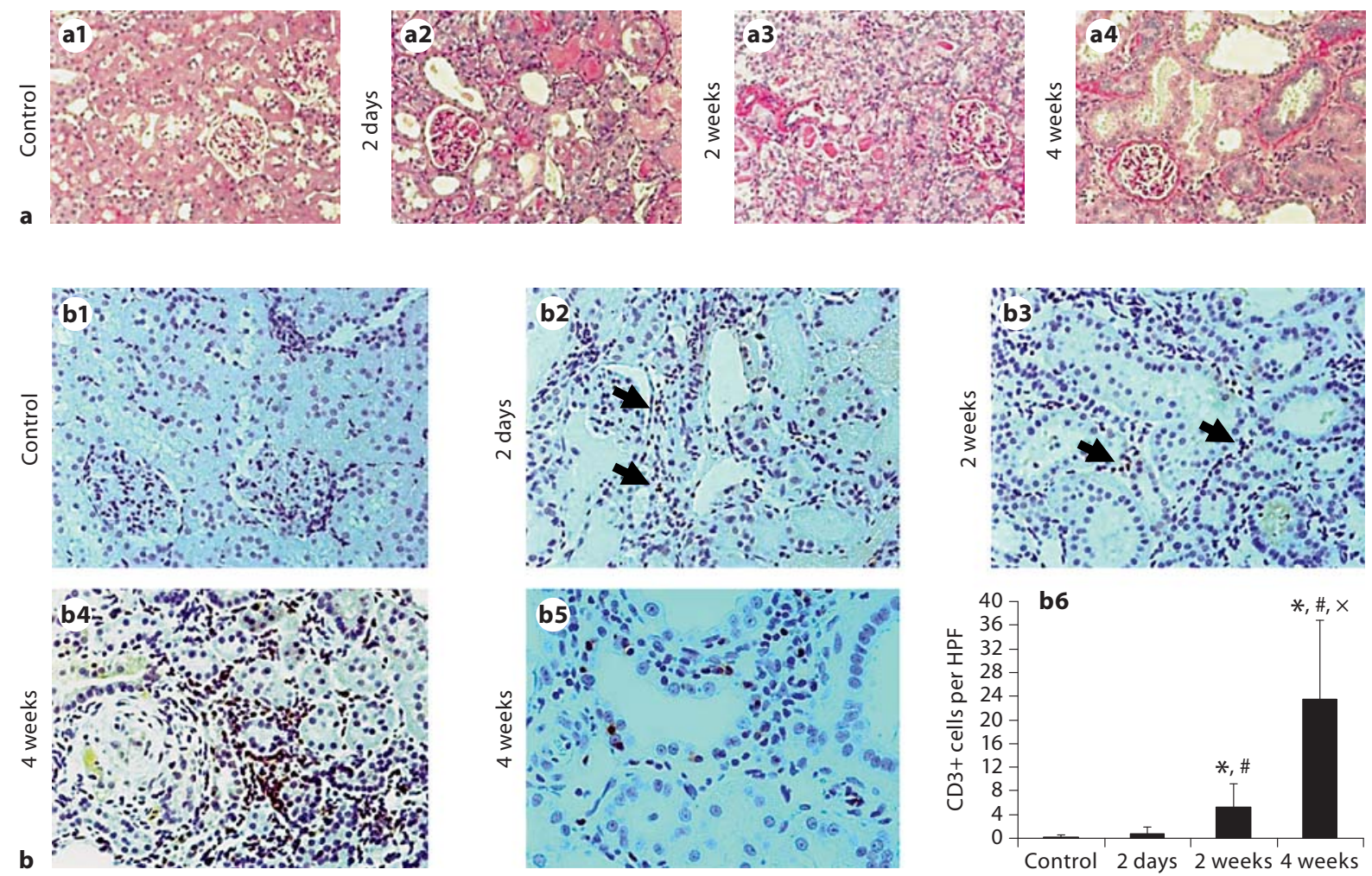

Fig. 2. Progressive tubulointerstitial injury and interstitial accumulation of CD3+ T lymphocytes in RK occurred from day 2 to week 4. a PAS staining of control (a1), 2 days (a2), 2 weeks (a3), and 4 weeks (a4) after 5/6Nx are shown. Control kidneys showed no histomorphologic changes. Two days after $5 / 6 \mathrm{Nx}$, there was extensive ischemic injury, with prominent interstitial inflammatory cell infiltration. At 2 weeks, the RK hypertrophied, with continued tubulointerstitial inflammation. By 4 weeks, marked glomerulosclerosis, tubular dilation with flattened tubular epithelial cells, and atrophy was present in the cortex of RK, which was paralleled by expansion of the interstitial space with the accumulation of interstitial cells. b Tissue sections were immunostained with a polyclonal rabbit anti-CD3+ antibody. Positive staining for $\mathrm{CD} 3+$ protein is shown in brown. All sections were counterstained with hematoxylin. In RK tissues, changes were characterized by the progressive accumulation of interstitial CD3+ T lymphocytes from day 2 to week 4 . There was evidence of tubulitis and interstitial CD3+ T lymphocytes in the RK at week 4 . b6 Differences between RK and control kidneys were significant for CD3+ T lymphocytes at weeks 2 and 4 . Values are given as mean \pm SD from three RK and three control kidneys at each time point. ${ }^{*} \mathrm{p}<0.01$ vs. control group, ${ }^{*} \mathrm{p}<0.01$ vs. 2 days after $5 / 6 \mathrm{Nx}$ group, ${ }^{\times} \mathrm{p}<0.01$ vs. 2 weeks after $5 / 6 \mathrm{Nx}$ group (a1-4, b1-4, orig. magnif. $\times 200$; b5, orig. magnif. $\times 400)$. vs. $0.2 \pm 0.4$ cells/HPF in the control kidneys, $\mathrm{p}<0.01)$ (fig. 2b).

\section{Ox-LDL Is Expressed in the Injured RK after $5 / 6 N x$}

There was minimal staining for Ox-LDL in normal kidney, while prominent Ox-LDL was identified diffusely in all RK. As shown in figure $3 \mathrm{a}$, at all time points, RK staining demonstrated Ox-LDL expression predominantly in tubular cells. The intensity of the tubular cell staining increased over time after $5 / 6 \mathrm{Nx}$. These experiments demonstrate the de novo accumulation of Ox-LDL in the injured RK after $5 / 6 \mathrm{Nx}$.

\section{Lectin-Like Ox-LDL Receptor (LOX-1) Expression after 5/6Nx Is Associated with the Appearance of $O x-L D L$ in the Renal Tubules}

Serial sections of the RK and control kidneys were stained for Ox-LDL and LOX-1, a receptor that internal- 


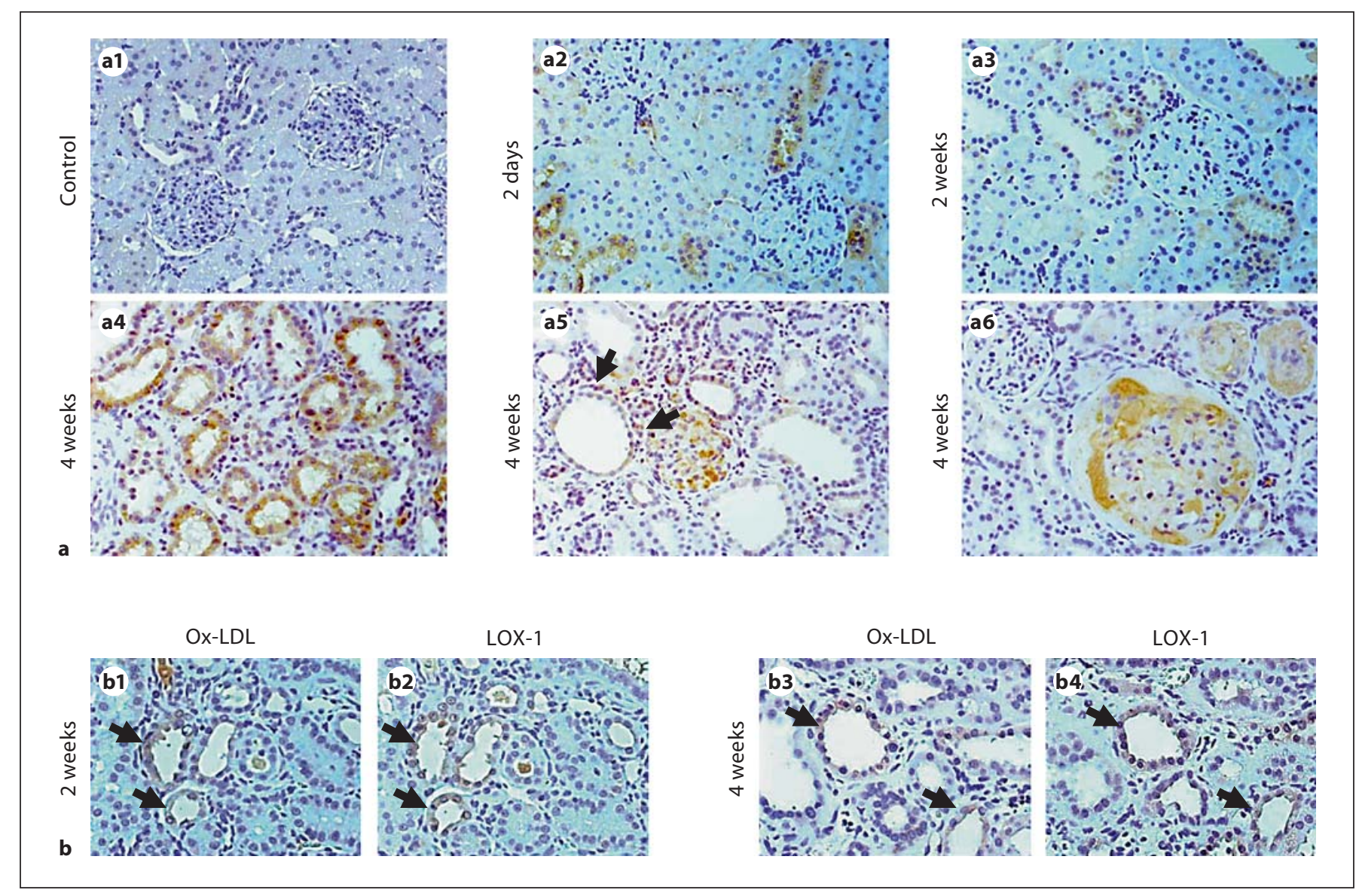

Fig. 3. Ox-LDL co-localizes with its receptor in the RK tissues after $5 / 6 \mathrm{Nx}$. a Tissue sections were immunostained with a monoclonal antibody (EO6). Positive staining for Ox-LDL protein is shown in brown. All sections were counterstained with hematoxylin. a1 There was minimal staining for Ox-LDL in normal kidney. In contrast, representative sections of kidney tissues at 2 days (a2), 2 weeks (a3), and 4 weeks (a4) display cytoplasmic staining for $\mathrm{Ox}-\mathrm{LDL}$, with stronger and more diffuse staining in tubular cells at 2 and 4 weeks. a5, a6 Localized heavy staining for Ox-LDL protein is shown in the interstitial areas (arrow) with lesions of glomerulosclerosis at 4 weeks after $5 / 6 \mathrm{Nx}$ (orig. magnif. $\times 200)$. b Serial sections of RK were stained for Ox-LDL (b1, b3) and LOX-1 (b2, b4) at 2 weeks (b1, b2), and 4 weeks (b3, b4) after $5 / 6 \mathrm{Nx}$. Staining of Ox-LDL showed co-localization with LOX-1 in renal tubular epithelium after $5 / 6 \mathrm{Nx}$ (arrow) (orig. magnif. $\times 200)$. izes oxidized lipids. To study the relationship of Ox-LDL and one of its major uptake receptors, tissues were stained for LOX-1, a type II single-pass membrane protein that binds and internalizes Ox-LDL. These immunohistochemical serial section studies reveal co-staining of $\mathrm{Ox}$ LDL with LOX-1 in affected renal tubular cells at all time points after $5 / 6 \mathrm{Nx}$ (fig. 3b). Thus, these studies, which demonstrate an association between Ox-LDL accumulation and the appearance of LOX-1 proteins in renal tubules, may implicate renal tubular Ox-LDL accumulation in the development of tubulointerstitial damage and progressive renal injury.

Ox-LDL Induces Renal Autoimmunity

\section{Renal-Specific Antigens and Novel Ox-LDL Are \\ Identified in RLNs after $5 / 6 \mathrm{Nx}$}

These experiments were performed to formally test whether migratory APCs from the kidney are capable of taking up and transporting renal-specific antigen and renal neoantigen from the inflamed kidney to RLNs. RLNs were removed 2 days, 2 weeks, and 4 weeks after $5 / 6 \mathrm{Nx}$ and stained for the kidney-specific intrinsic glycoprotein THP and the posited de novo renal neoantigen Ox-LDL (fig. 4a, b). These experiments identify both neoantigen and renal-specific intrinsic antigen expression in the RLNs after $5 / 6 \mathrm{Nx}$. These findings suggest that the tubulointerstitial inflammatory milieu arising as a conse- 

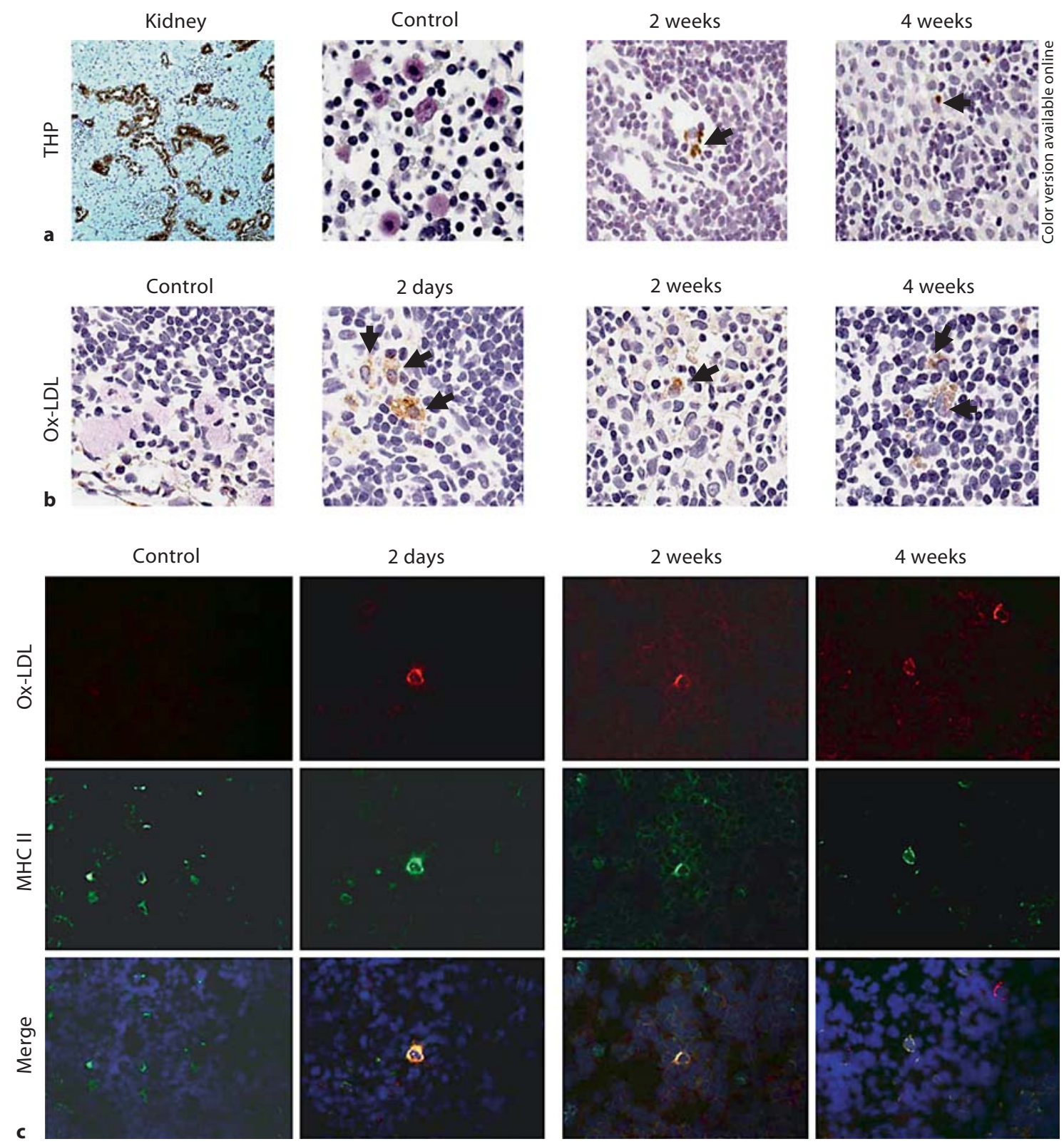

Fig. 4. De novo expression of renal-specific THP antigen and OxLDL that co-localizes with APC marker in the RLNs after $5 / 6 \mathrm{Nx}$. a Representative photomicrographs of kidney (positive control, orig. magnif. $\times 100$ ), and RLN sections of controls, $5 / 6 \mathrm{Nx}$ at 2 weeks and 5/6Nx at 4 weeks (orig. magnif. $\times 400$ ) stained for THP. THP was not detected in RLN sections of controls, but representative sections of RLN at 2 and 4 weeks display cytoplasmic staining for THP (arrow). b Representative photomicrographs of Ox-LDL-stained RLN sections of controls, and 5/6Nx at 2 days, 2 weeks and 4 weeks. Ox-LDL was not detected in RLN sections of controls, but representative sections of RLN cells at 2 days, 2 weeks, and 4 weeks display cytoplasmic staining for Ox-LDL (arrow) (orig. magnif. $\times 400$ ). c The sections show immunofluorescence staining patterns for Ox-LDL (red) and MHC II (green) in RLN tissues 2 days, 2 weeks and 4 weeks after $5 / 6 \mathrm{Nx}$. The sections were counterstained with DAPI to visualize cell nuclei (merge). Ox-LDL (red) staining was detected in the RLNs (upper panel). Staining of Ox-LDL (red) co-localized with a subset of MHC class II-positive cells (green) in the RLNs at 2 days, 2 weeks and 4 weeks after 5/6Nx (orig. magnif. $\times 400$ ). 
quence of 5/6Nx may transform self-proteins into neoantigens and stimulate the trafficking of endogenous renal antigens from the kidney to draining lymphoid tissue on a continuous basis during the evolution of renal injury.

\section{APCs Carrying Antigens from the Kidney Egress via} Lymphatics after Renal Injury

This additional experiment tested whether localized inflammatory events in the kidney occurring after $5 / 6 \mathrm{Nx}$ initiates efflux of APCs from the renal parenchyma to the RLNs. To test this hypothesis, RLNs were double stained by immunofluorescence for APC markers (MHC class II), and for the de novo expression of Ox-LDL after $5 / 6 \mathrm{Nx}$. Cells co-staining for MHC class II and Ox-LDL were observed in the draining RLNs, albeit few in number, at all times after $5 / 6 \mathrm{Nx}$ (fig. 4c).

\section{Ox-LDL-Treated APCs Activate T Cells Isolated from Injured Tubulointerstitium}

The available renal mass after $5 / 6 \mathrm{Nx}$ is a limitation for certain kinds of experiments. In order to test the generalizability of the phenomenon of neoantigen generation in injured kidney, and to perform T-lymphocyte isolation from a greater renal mass, these experiments were performed on $\mathrm{T}$ lymphocytes isolated from kidneys after UUO. UUO resulted in a significant and progressive increase in interstitial lymphocyte infiltration in all obstructed kidneys compared with intact opposite kidneys (fig. 5a). At 2 weeks, we also demonstrated more intense Ox-LDL expression, predominantly in tubular cells of UUO kidneys compared with control kidneys (fig. 5b). RAW264.7 cells are a transformed murine macrophage cell line capable of a phenotypic change into dendritic-like APCs when incubated with Ox-LDL [14]. As shown in figure 5c, Ox-LDL exposure upregulated MHC II expression on the surface of RAW264.7 cells. These data suggest that Ox-LDL induced differentiation of RAW264.7 cells into APCs. RAW264.7 cells were incubated with Ox-LDL for $48 \mathrm{~h}$ and then cultured with purified renal tubulointerstitial or splenic T cells for 5 days. The RAW264.7 cells treated with 10 and $20 \mu \mathrm{g} / \mathrm{ml}$ Ox-LDL effectively induced proliferation of renal tubulointerstitial T cells, but not splenic T cells, by 1.6 - to 1.8 -fold (fig. $5 \mathrm{~d}$ ). As figure 5 e shows, $20 \mu \mathrm{g} / \mathrm{ml}$ of Ox-LDL-treated RAW264.7 cells also significantly stimulated IFN- $\gamma$ secretion by renal tubulointerstitial $\mathrm{T}$ cells. Identical co-cultures performed with $\mathrm{T}$ cells from the spleen of UUO mice did not secrete significant amounts of IFN- $\gamma$ (data not shown). These results show that $\mathrm{T}$ lymphocytes derived from injured tubulointerstitium demonstrate pre-sensitization to Ox-LDL antigen.

\section{Discussion}

These studies demonstrate that the exogenous lipoprotein Ox-LDL (and the endogenous protein THP), as potential renal-derived antigens, are transported by APCs from injured kidney to draining RLNs; elucidate the incremental expression of tubulointerstitial chemokine ligand-receptor pairs which facilitate the entry of infiltrating leukocytes in the tubulointerstitial area, and demonstrate that Ox-LDL-treated MHC II-expressing APCs regulate the proliferation and cytokine production of $\mathrm{T}$ lymphocytes derived from the injured tubulointerstitium. Taken together, these studies suggest that the inflammatory milieu that accompanies renal injury may transform native proteins into neoantigens, inducing interstitial autoimmunity. Autoimmunity to altered native renal antigens may foster continued tubulointerstitial damage, independent of the initial inciting injury.

The efflux of mature APCs to draining lymphoid organs and the recruitment of $\mathrm{T}$ lymphocytes to regions in the inflamed kidneys are mediated by inflammatory chemokines [15]. CXCR3 is a chemokine receptor that is highly expressed on T lymphocytes. In a variety of human renal inflammatory diseases, including glomerulonephritis [16] and acute transplant rejection [17], the enhanced expression of CXCR3 chemokine ligands is followed by the recruitment of $\mathrm{T}$ lymphocytes. The inflammation that triggers APC migration through lymphatic vessels requires the chemokine receptor CCR7 and its ligands $[13,18]$. In inflammatory kidney diseases, CCR7-bearing mature APCs may leave the renal tissue via CCL19/CCL21-positive lymphatic endothelial cells to present their antigen in secondary lymphatic organs [18]. Here we show that in experimental CKD, the injured kidney produces CXCR3/CXCL10 and CCL19/CCR7 mRNA, providing evidence for CXCL10-mediated lymphocyte infiltration and presumably also CCL19-mediated APC chemoattraction by lymphatic vessels. These findings support APC-mediated trafficking from renal injured kidneys to draining lymph nodes.

Renal lipid accumulation aggravates glomerular and tubulointerstitial injury in a variety of experimental renal diseases $[19,20]$. Interestingly, our study showed that in the setting of chronic tubulointerstitial injury, Ox-LDL enhanced renal T-cell proliferation and induced IFN- $\gamma$ secretion, which is produced by $\mathrm{T}$ cells. These findings are consistent with published evidence that Ox-LDL exposure favors monocyte differentiation [14, 21], and activates $\mathrm{T}$ cells by an MHC II-dependent mechanism [11]. Thus, our data are consistent with the hypothesis that 


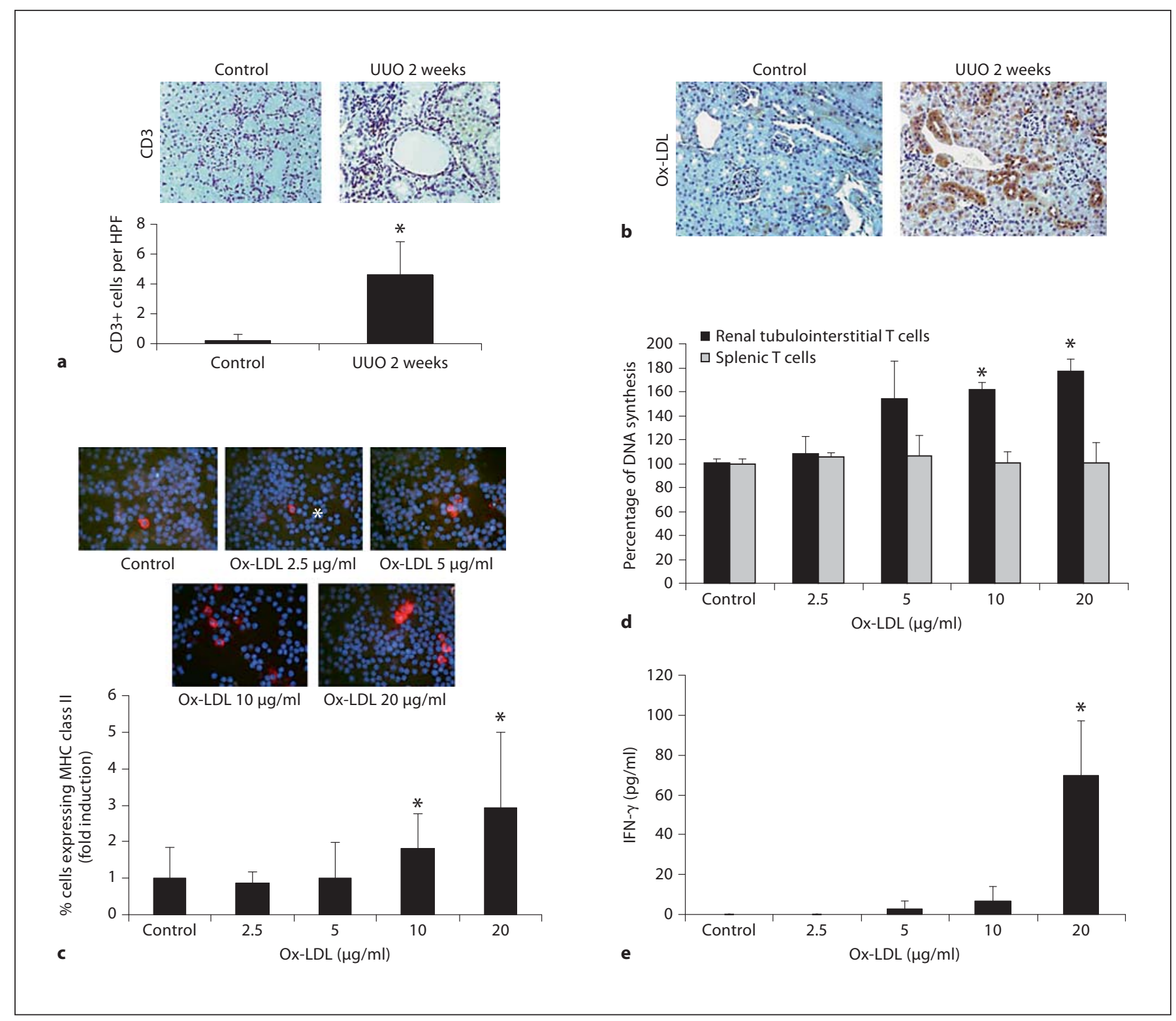

Fig. 5. T cells isolated from UUO kidneys with tubulointerstitial injury are stimulated by OX-LDL-treated APCs. a Representative photomicrographs illustrating CD3+ lymphocytes (brown staining) in UUO kidney at 2 weeks and control kidney. Differences between UUO and control kidneys were significant for CD3+ T lymphocytes at week 2 . Values are given as mean $\pm S D .{ }^{*} \mathrm{p}<0.01$ vs. control group. b Representative photomicrographs of $\mathrm{Ox}$ LDL-stained kidney sections of control and UUO at 2 weeks. OxLDL was slightly detected in controls. In contrast, representative sections of kidney tissues at 2 weeks displayed cytoplasmic staining for Ox-LDL, most prominently in the renal tubular cells, with stronger and more diffuse tubular cell staining at 4 weeks (orig. magnif. $\times 200$ ). c Representative photomicrographs illustrating MHC II (red staining) in RAW264.7 cells untreated or treated with Ox-LDL. Quantitative analysis of cells showing MHC II in distinct locations demonstrated that the percentages of MHC IIpositive RAW264.7 cells increased significantly after 10 and 20 $\mu \mathrm{g} / \mathrm{ml}$ of Ox-LDL treatment. ${ }^{*} \mathrm{p}<0.05$ vs. control group. d T-cell proliferation was activated by Ox-LDL-treated RAW264.7 cells. RAW264.7 cells were cultured for $48 \mathrm{~h}$ in the absence or presence of varying $\mathrm{Ox}-\mathrm{LDL}$ doses. Activation was performed at a ratio of 1:10 (RAW264.7:T cells) for 5 days and $20 \mu$ l diluted BrdU label was added to the appropriate wells. RAW264.7 cells treated with 10 or $20 \mu \mathrm{g} / \mathrm{ml}$ Ox-LDL significantly stimulated proliferation of $\mathrm{T}$ cells isolated from the tubulointerstitium of kidneys after injury induced by UUO, but no changes were observed in T cells from the spleen. ${ }^{*} \mathrm{p}<0.01$ vs. control group. e Ox-LDL-treated RAW264.7 cells stimulated IFN- $\gamma$ secretion by renal tubulointerstitial T cells. Supernatants from co-culture of Ox-LDL-treated RAW264.7 cells and renal T cells were collected at day 5 and the amount of IFN- $\gamma$ was measured by sandwich ELISA. Significant changes in IFN- $\gamma$ production were observed in culture supernatants of $20 \mu \mathrm{g} / \mathrm{ml}$ Ox-LDL-treated RAW264.7 cells with T cells. ${ }^{*} \mathrm{p}<0.05$ vs. control group. 
Ox-LDL acts as an endogenous stimulus inducing a Tcell-dependent immune reaction, which may play an important role in the development of progressive renal injury through local tubulointerstitial replication and the development of interstitial nephritis.

Renal APCs, herein defined on the basis of MHC class II expression, reside within the renal parenchyma and are crucial for inducing and regulating adaptive immunity $[22,23]$. Interactions between $\mathrm{T}$ lymphocytes and renal APCs occur following experimental renal injury, inducing T-cell antigen specificity for renal antigens in local lymph nodes. In addition, after the acute kidney injury (AKI) induced by lipopolysaccharide injection, THP was identified in RLNs expressed on APCs [5]. These antigen presentation events occurring during AKI are potentially injurious to the kidney [24, 25]. Recently, in $5 / 6 \mathrm{Nx}$ and other CKD models, it has also been shown that filterable molecules such as albumin are not only taken up by tubular epithelial cells, but also by renal APCs, where they are transported by these cells to RLNs, and induce immunity [3]. Our work extends these observations in two ways. Firstly, our experiments show that THP is transported to RLNs by APCs not only in a model of AKI, but also in experimental CKD. Secondly, our studies show that albumin is not the only potentially immunogenic neoantigen derived from the injured kidney, as Ox-LDL also traffics to the RLNs on APCs. Thus, our study in the RK and UUO models provide clear evidence that chronic kidney injury is also associated with abnormal APC-mediated passage of renal antigens to the RLNs.

This study has a number of limitations. One limitation is that the renal $\mathrm{T}$ cells that were isolated from the UUO kidney to demonstrate Ox-LDL autoreactivity were isolated using a chemical digestion step that was not necessary for the isolation of splenic T cells, leaving open the possibility that the differences observed were due to technical handling differences and not to differences in antigen recognition per se. Secondly, at the time points studied, only a small number of APCs co-localizing MHC II and Ox-LDL were identified. However, the magnitude of expression herein is similar to that observed for THP after lipopolysaccharide exposure [5]. Finally, these observations were made at relatively early time points in the evolution of experimental CKD. The well-known persistence of a tubulointerstitial mononuclear infiltrate accompanying most chronic renal disease suggests that this process persists. However, direct observations at later time points and intervention would be required to demonstrate chronicity and causation.
In conclusion, this study demonstrates endogenous and exogenous renal antigen transport by APCs to draining lymph nodes from $\mathrm{RK}$ after $5 / 6 \mathrm{Nx}$. It also identifies chemokine ligand-receptor pairs that facilitate dendritic cell trafficking away from the kidney and the return of activated $\mathrm{T}$ lymphocytes to the inflamed renal tissue. Finally, it demonstrates that $\mathrm{T}$ cells isolated from injured renal tubulointerstitium demonstrate pre-sensitization to Ox-LDL by proliferating and secreting cytokine. Taken together, this study shows that the early tubulointerstitial inflammatory infiltrate that accompanies CKD reflects, at least in part, the development of autoimmunity to novel antigens generated during renal injury, suggesting that interventions directed at dendritic cell and T-lymphocyte activation and trafficking may be fertile targets for the attenuation of progressive kidney disease.

\section{Acknowledgments}

We are grateful for support from the National Kidney Foundation of Southern California (B.S.), the International Society of Nephrology (B.S.), the National Kidney Foundation of Thailand (B.S.), and the National Institute of Diabetes and Digestive and Kidney Diseases (R.N. and S.G.A.). The authors thank Dr. Alan Fogelman and Dr. Michael Yeaman for helpful discussions. This work was funded in part by a DaVita research grant.

\section{Disclosure Statement}

The authors have no conflicts of interest to disclose.

\section{References}

\footnotetext{
1 Drayton DL, Liao S, Mounzer RH, Ruddle $\mathrm{NH}$ : Lymphoid organ development: from ontogeny to neogenesis. Nat Immunol 2006; 7:344-353.

2 Mellman I, Steinman RM: Dendritic cells: specialized and regulated antigen processing machines. Cell 2001;106:255-258.

-3 Macconi D, Chiabrando C, Schiarea S, Aiello S, Cassis L, Gagliardini E, Noris M, Buelli S, Zoja C, Corna D, Mele C, Fanelli R, Remuzzi $\mathrm{G}$, Benigni A: Proteasomal processing of albumin by renal dendritic cells generates antigenic peptides. J Am Soc Nephrol 2009;20: 123-130.

-4 Heymann F, Meyer-Schwesinger C, Hamilton-Williams EE, Hammerich L, Panzer U, Kaden S, Quaggin SE, Floege J, Grone HJ, Kurts C: Kidney dendritic cell activation is required for progression of renal disease in a mouse model of glomerular injury. J Clin Invest 2009;119:1286-1297.
} 
5 Dong X, Swaminathan S, Bachman LA, Croatt AJ, Nath KA, Griffin MD: Antigen presentation by dendritic cells in renal lymph nodes is linked to systemic and local injury to the kidney. Kidney Int 2005;68: 1096-1108.

-6 Kwan BC, Kronenberg F, Beddhu S, Cheung AK: Lipoprotein metabolism and lipid management in chronic kidney disease. J Am Soc Nephrol 2007;18:1246-1261.

$\checkmark 7$ Wheeler DC, Chana RS, Topley N, Petersen MM, Davies M, Williams JD: Oxidation of low density lipoprotein by mesangial cells may promote glomerular injury. Kidney Int 1994;45:1628-1636.

8 Solin ML, Ahola H, Haltia A, Ursini F, Montine T, Roveri A, Kerjaschki D, Holthofer H: Lipid peroxidation in human proteinuric disease. Kidney Int 2001;59:481-487.

$\checkmark 9$ Song CY, Kim BC, Hong HK, Lee HS: Oxidized LDL activates PAI-1 transcription through autocrine activation of TGF- $\beta$ signaling in mesangial cells. Kidney Int 2005; 67:1743-1752.

$>10$ Fortun A, Khalil A, Gagne D, Douziech N, Kuntz C, Jay-Gerin JP, Dupuis G, Fulop T Jr: Monocytes influence the fate of T cells challenged with oxidised low density lipoproteins towards apoptosis or MHC-restricted proliferation. Atherosclerosis 2001;156:1121.

-11 Huang YH, Ronnelid J, Frostegard J: Oxidized LDL induces enhanced antibody formation and MHC class II-dependent IFN- $\gamma$ production in lymphocytes from healthy individuals. Arterioscler Thromb Vasc Biol 1995; 15:1577-1583.
2 Kim HJ, Moradi H, Yuan J, Norris K, Vaziri ND: Renal mass reduction results in accumulation of lipids and dysregulation of lipid regulatory proteins in the remnant kidney. Am J Physiol Renal Physiol 2009;296:F1297F1306.

13 Jang MH, Sougawa N, Tanaka T, Hirata T, Hiroi T, Tohya K, Guo Z, Umemoto E, Ebisuno Y, Yang BG, Seoh JY, Lipp M, Kiyono H, Miyasaka M: CCR7 is critically important for migration of dendritic cells in intestinal lamina propria to mesenteric lymph nodes. J Immunol 2006;176:803-810.

14 Shen LH, Zhou L, Wang BY, Pu J, Hu LH, Chai DJ, Wang L, Zeng JZ, He B: Oxidized low-density lipoprotein induces differentiation of RAW264.7 murine macrophage cell line into dendritic-like cells. Atherosclerosis 2008;199:257-264.

15 Anders HJ, Vielhauer V, Schlondorff D: Chemokines and chemokine receptors are involved in the resolution or progression of renal disease. Kidney Int 2003;63:401-415.

- 16 Segerer S, Banas B, Wornle M, Schmid H, Cohen CD, Kretzler M, Mack M, Kiss E, Nelson PJ, Schlondorff D, Grone HJ: CXCR3 is involved in tubulointerstitial injury in human glomerulonephritis. Am J Pathol 2004; 164:635-649.

17 Panzer U, Reinking RR, Steinmetz OM, Zahner G, Sudbeck U, Fehr S, Pfalzer B, Schneider A, Thaiss F, Mack M, Conrad S, Huland H, Helmchen U, Stahl RA: CXCR3 and CCR5 positive T-cell recruitment in acute human renal allograft rejection. Transplantation 2004;78:1341-1350.

18 Steinmetz OM, Stahl RA, Panzer U: Formation of lymphoid-like tissue in the kidney - is there a role for chemokines? Nephrol Dial Transplant 2007;22:350-352.
19 Lee HS, Jeong JY, Kim BC, Kim YS, Zhang YZ, Chung HK: Dietary antioxidant inhibits lipoprotein oxidation and renal injury in experimental focal segmental glomerulosclerosis. Kidney Int 1997;51:1151-1159.

-20 Buga GM, Frank JS, Mottino GA, Hakhamian A, Narasimha A, Watson AD, Yekta B, Navab M, Reddy ST, Anantharamaiah GM, Fogelman AM: D-4F reduces EO6 immunoreactivity, SREBP-1c MRNA levels, and renal inflammation in LDL receptor-null mice fed a Western diet. J Lipid Res 2008;49:192-205.

-21 Perrin-Cocon L, Coutant F, Agaugue S, Deforges S, Andre P, Lotteau V: Oxidized lowdensity lipoprotein promotes mature dendritic cell transition from differentiating monocyte. J Immunol 2001;167:3785-3791.

22 Soos TJ, Sims TN, Barisoni L, Lin K, Littman DR, Dustin ML, Nelson PJ: CX3CR1+ interstitial dendritic cells form a contiguous network throughout the entire kidney. Kidney Int 2006;70:591-596.

-23 Rogers NM, Matthews TJ, Kausman JY, Kitching RA, Coates PT: Kidney dendritic cells: their role in homeostasis, inflammation and transplantation. Nephrology (Carlton) 2009; 14:625-635.

24 Chandraker A, Takada M, Nadeau KC, Peach R, Tilney NL, Sayegh MH: CD28-B7 blockade in organ dysfunction secondary to cold ischemia/reperfusion injury. Kidney Int 1997;52:1678-1684.

25 Yokota N, Daniels F, Crosson J, Rabb H: Protective effect of T-cell depletion in murine renal ischemia-reperfusion injury. Transplantation 2002;74:759-763. 